\title{
Stimulated Hawking emission from electromagnetic analogue black hole: Theory and observation
}

\author{
Avijit Bera ${ }^{*}$ and Subir Ghosh \\ Physics and Applied Mathematics Unit, Indian Statistical Institute, Kolkata-700108, India
}

(Received 10 February 2020; accepted 20 April 2020; published 15 May 2020)

\begin{abstract}
In this paper we consider possible analogue Hawking radiation from a normal dielectric and metamaterial composite, having an analogue horizon where the dielectric parameters vanish and change sign upon crossing this transition zone. We follow a complex path analysis to show the presence of an analogue Hawking temperature at the horizon and subsequent photon production from the ambient electromagnetic field. The possibility of experimental observation is also commented upon.
\end{abstract}

DOI: 10.1103/PhysRevD.101.105012

\section{INTRODUCTION}

Even after around 45 years, Hawking's theoretical discovery [1] of black hole $(\mathrm{BH})$ radiation remains in the limelight in a somewhat enigmatic way since no smoking gun evidence of it has been produced in the astrophysical context, the major reason being the tiny value of Hawking temperature $\sim 10^{-8} \mathrm{~K}$ for a solar mass black hole. This has led to a flurry of activity in the analogue gravity scenario, pioneered by Unruh [2] in a fluid system, followed by many works in different physical systems (see [3] for a review). In all these cases the underlying principle is that the relevant degree of freedom of a physical system (that need not have any direct connection to general relativity or gravitation and preferably possible to construct the system in a laboratory) satisfies an equation of motion that can be cast in the conventional form of dynamics in an effective curved spacetime. This allows the identification of an effective metric constructed out of parameters of the analogue system. However, an analogue black hole and subsequently analogue Hawking radiation (HR) is possible only if the effective metric has a "horizon" where the metric coefficients vanish or become singular, as the case may be. The "dumb hole" theorized by Unruh [2] and later experimentally constructed and studied by [4] occurred in a moving fluid (with a critical velocity) that acoustic disturbance cannot penetrate. (In fact the experimental setups are more geared to an analogue white hole).

\footnotetext{
*avijitbera1997@gmail.com

†subirghosh20@gmail.com
}

Published by the American Physical Society under the terms of the Creative Commons Attribution 4.0 International license. Further distribution of this work must maintain attribution to the author(s) and the published article's title, journal citation, and DOI. Funded by SCOAP.
Let us come to an optical analogue of $\mathrm{BH}$, that is of present interest. It has long been well known [5] (in purely classical framework) that electrodynamics in curved spacetime can be mapped to electrodynamics in flat spacetime in a dielectric medium with nonuniform permittivity and permeability. This theme received a strong impetus in recent times after the laboratory construction of an exotic form of dielectric - metamaterial, with negative permittivity and permeability. The possible existence of metamaterial was visualized long ago by Veselago [6] and its experimental verification was done in [7]. Its counterintuitive properties have led to many significant applications such as negative refraction, electromagnetic invisibility cloaks, superresolution imaging, electromagnetic concentrators and light trapping, among others [8]. The essential features of the constitutive parameters can be induced in periodic or nonperiodic arrays of electric or magnetic resonant or nonresonant particles. Electrodynamics in medium with both normal and metamaterial features has become a popular playground for theorists since exploiting the above curved spacetimedielectric mapping the latter can serve as analogue models of exotic forms of spacetime [9].

Specific theoretical suggestion and possible construction of an omnidirectional electromagnetic absorber in the microwave frequency that can serve as an optical analogue $\mathrm{BH}$ appeared in [10]. Laboratory construction $[11,12]$ of a graded metamaterial disk with rotational symmetry has demonstrated $\mathrm{BH}$-like features by absorbing incident radiation extremely efficiently. The system, with a metamaterial core inside, can trap and absorb electromagnetic waves coming from all directions that spiral inwards without any reflections. The theoretical study (and the subsequent experiment) is based on a purely classical analysis of the Maxwell equations in a nonuniform dielectric medium. We emphasize that this is an optical analogue of a classical $\mathrm{BH}$ and so analogue Hawking effect, a quantum 
phenomenon, has not been considered. An attempt of metamaterial $\mathrm{BH}$ with possible Hawking effect is given in [13]. In an earlier work [14] a similar idea was put forward without explicitly using the metamaterial framework. In a dynamical system, using a perturbative quantum field theory framework, the analogue Hawking effect was predicted by [15] and experimentally observed by [16] where the nonlinear Kerr effect was induced in the medium by a laser pulse.

In this paper, in a completely novel analysis, we show that the model of an optical analogue $\mathrm{BH}$ proposed and constructed in [10-12] can generate stimulated HR. The difference between the two is that in BH HR [1] is emitted spontaneously at the cost of lowering the $\mathrm{BH}$ mass whereas in stimulated HR the source of analogue HR is the ambient electromagnetic field. The dielectricmetamaterial interface creates an effective horizon that allows emergence of photons only whereas in BH HR all forms of particles are allowed to emerge. We have gone beyond the classical analysis [10] to a semiclassical analysis where quantum tunnelings are involved. Precisely the latter are taken care of by introducing the complex path formalism [17].

The original work of Hawking used Bogoliubov coefficients but later identical results have been recovered in different frameworks such as tunneling [18] picture, anomaly [19], Hamilton-Jacobi (HJ) with complex path [20]. This clearly underlines the universality of the HR phenomenon. We will use the HJ approach initiated by Srinivasan and Padmanabhan [20] because of its generality. Generally in HR (analogue HR) one deals with the field equation in curved metric (effective metric) respectively that has horizon (effective horizon). We, on the other hand, will start directly from electromagnetic wave equation, derived from Maxwell's equations, and show that a horizonlike property of the dielectric parameters can induce an analogue Hawking temperature and analogue HR since the solution of the HJ equation has an imaginary part developed in the transition zone.

The paper is organized as follows. In Sec. II we discuss electrodynamics in (spherically symmetric) nonuniform dielectric medium. In Sec. III we develop the HamiltonJacobi formalism for the specific form of normalmetamaterial composite. Section IV deals briefly with the analogue Schrödinger problem. Our main results are provided in Sec. V where we derive the analogue Hawking temperature for the graded metamaterial composite and suggest its possible experimental observation. The papers ends with a discussion in Sec. VI.

\section{ELECTRODYNAMICS IN NONUNIFORM MEDIUM}

Maxwell's equations in dielectric medium free of charge and current density are given by

$$
\begin{aligned}
& \nabla \cdot \mathbf{D}=0, \quad \nabla \cdot \mathbf{B}=0 \\
& \nabla \times \mathbf{E}=-\frac{\partial \mathbf{B}}{\partial t}, \quad \nabla \times \mathbf{H}=-\frac{\partial \mathbf{D}}{\partial t} .
\end{aligned}
$$

In spherical polar coordinates $r, \theta, \phi$ let us define the displacement vector $\mathbf{D}$ and magnetic induction $\mathbf{B}$ from the electric field $\mathbf{E}$ and magnetic field $\mathbf{H}$ as

$$
\begin{array}{ccc}
D_{r}=\epsilon_{1} E_{r}, & D_{\theta}=\epsilon_{2} E_{\theta}, & D_{\phi}=\epsilon_{2} E_{\phi} \\
B_{r}=\mu_{1} H_{r}, & B_{\theta}=\mu_{2} H_{\theta}, & B_{\phi}=\mu_{2} H_{\phi},
\end{array}
$$

where we restrict to $\epsilon_{i}(r), \mu_{i}(r)$, i.e. permittivity $\epsilon$ and permeability $\mu$ are both the functions of radial distance $r$ only. This yields

$$
\begin{aligned}
\nabla \cdot \mathbf{E}= & -\frac{E_{r}}{\epsilon_{1}} \partial_{r} \epsilon_{1}+(1-a) \frac{1}{r \sin \theta} \partial_{\theta}\left(\sin \theta E_{\theta}\right) \\
& +(1-b) \frac{1}{r \sin \theta} \partial_{\phi} E_{\phi},
\end{aligned}
$$

where $a=\frac{\epsilon_{2}}{\epsilon_{1}}$ and $b=\frac{\epsilon_{3}}{\epsilon_{1}}$. In a similar way we obtain, with $p=\frac{\mu_{2}}{\mu_{1}}, q=\frac{\mu_{3}}{\mu_{1}}$,

$$
\begin{aligned}
\nabla \cdot \mathbf{H}= & -\frac{H_{r}}{\mu_{1}} \partial_{r} \mu_{1}+(1-p) \frac{1}{r \sin \theta} \partial_{\theta}\left(\sin \theta H_{\theta}\right) \\
& +(1-q) \frac{1}{r \sin \theta} \partial_{\phi} H_{\phi} .
\end{aligned}
$$

For simplicity we restrict further to $a=b=1$ and also $p=q=1$ leading to

$\nabla \cdot \mathbf{E}=-\frac{1}{\epsilon(r)} \nabla \epsilon(r) \cdot \mathbf{E}, \quad \nabla \cdot \mathbf{H}=-\frac{1}{\mu(r)} \nabla \mu(r) \cdot \mathbf{H}$.

It is straightforward to recover the wave equation satisfied by $\mathbf{E}$ as (with a similar equation for $\mathbf{H}$ )

$\nabla^{2} \mathbf{E}-\mu \epsilon \frac{\partial^{2} \mathbf{E}}{\partial t^{2}}+\nabla\left(\frac{1}{\epsilon}\right)(\nabla \epsilon \cdot \mathbf{E})+\left(\frac{1}{\epsilon}\right) \nabla(\nabla \epsilon \cdot \mathbf{E})=0$.

Exploiting spherical symmetry of our system, we expand the electric field in terms of vector spherical harmonics:

$\mathbf{E}=\sum_{l=0}^{\infty} \sum_{m=0}^{\infty}\left[E_{l m}^{r}(r) \mathbf{Y}_{l m}+E_{l m}^{1}(r) \Psi_{l m}+E_{l m}^{2}(r) \Phi_{l m}\right]$,

where $E_{l m}^{r}(r)$ is the radial component and $E_{l m}^{1}(r), E_{l m}^{2}(r)$ are the transverse components of the electric field. The vector spherical harmonics are defined in terms of conventional spherical harmonics as 


$$
\begin{aligned}
& \mathbf{Y}_{l m}=Y_{l m} \hat{r} \\
& \Psi_{l m}=r \nabla Y_{l m}=\left[\hat{\theta} \frac{\partial}{\partial \theta}+\hat{\phi} \frac{1}{\sin \theta} \frac{\partial}{\partial \phi}\right] Y_{l m} \\
& \Phi_{l m}=\mathbf{r} \times \nabla Y_{l m}=\left[\hat{\phi} \frac{\partial}{\partial \theta}-\hat{\theta} \frac{1}{\sin \theta} \frac{\partial}{\partial \phi}\right] Y_{l m} .
\end{aligned}
$$

After a fair amount of messy algebra (some steps are given in Appendix B) we isolate wave equations of the individual components of $\mathbf{E}$,

$$
\begin{aligned}
& \frac{\partial^{2} E_{l m}^{r}}{\partial r^{2}}-\mu(r) \epsilon(r) \frac{\partial^{2} E_{l m}^{r}}{\partial t^{2}}+\left\{\frac{2}{r}+\frac{1}{\epsilon(r)} \frac{\partial \epsilon(r)}{\partial r}\right\} \frac{\partial E_{l m}^{r}}{\partial r} \\
& +\frac{l(l+1)}{r^{2}}\left(2 E_{l m}^{1}-E_{l m}^{r}\right) \\
& +\left\{\frac{\partial}{\partial r}\left(\frac{1}{\epsilon(r)} \frac{\partial \epsilon(r)}{\partial r}\right)-\frac{2}{r^{2}}\right\} E_{l m}^{r}=0 \\
& {\left[\frac{1}{r^{2}}\left\{\frac{\partial}{\partial r}\left(r^{2} \frac{\partial E_{l m}^{1}}{\partial r}\right)-l(l+1) E_{l m}^{1}\right\}-\frac{E_{l m}^{1}}{r^{2} \sin ^{2} \theta}\right.} \\
& \left.-\mu(r) \epsilon(r) \frac{\partial^{2} E_{l m}^{1}}{\partial t^{2}}+\left\{\frac{2}{r^{2}}+\frac{1}{r \epsilon(r)} \frac{\partial \epsilon(r)}{\partial r}\right\} E_{l m}^{r}\right] \\
& \quad \times\left\{\sin \theta\left(\frac{\partial Y_{l m}}{\partial \theta}\right)^{2}+\frac{1}{\sin \theta}\left(\frac{\partial Y_{l m}}{\partial \phi}\right)^{2}\right\} \\
& -\frac{2 \cos \theta}{r^{2} \sin ^{2} \theta}\left[E_{l m}^{1} \frac{\partial Y_{l m}}{\partial \theta}+\frac{E_{l m}^{2}}{\sin \theta} \frac{\partial Y_{l m}}{\partial \phi}\right]=0 .
\end{aligned}
$$

There is a similar equation for $E_{l m}^{2}$ in Appendix B. Let us concentrate on the $s$-wave mode that is $l=m=0$ for which Eq. (9) identically vanish and the $E_{00}^{r} \equiv E$ equation [Eq. (8)] is considerably simplified,

$E^{\prime \prime}-\mu \epsilon \frac{\partial^{2} E}{\partial t^{2}}+\left\{\frac{2}{r}+\frac{\epsilon^{\prime}}{\epsilon}\right\} E^{\prime}+\left\{\left(\frac{\epsilon^{\prime}}{\epsilon}\right)^{\prime}-\frac{2}{r^{2}}\right\} E=0$,

where ()$^{\prime} \equiv \partial_{r}()$.

As an aside we mention an intriguing result: how will the wave behave in a medium whose dielectric parameters are obtained from the Schwarzschild BH metric

$$
\begin{aligned}
d S^{2}= & g_{\mu \nu} d x^{\mu} d x^{\nu}=\left(1-\frac{R}{r}\right) d t^{2}+\frac{d r^{2}}{\left(1-\frac{R}{r}\right)} \\
& +r^{2}\left(d \theta^{2}+\sin ^{2} \theta d \phi^{2}\right)
\end{aligned}
$$

through the mapping [5]

$$
\epsilon^{i j}=\mu^{i j}=-\frac{\sqrt{-g}}{g_{00}} g^{i j} .
$$

Since the mapping is in the Cartesian coordinate system the corresponding parameters are explicitly written as $\left.\epsilon^{i j}\right|_{\text {Cart }}=\left.\mu^{i j}\right|_{\text {Cart }}=-\frac{1}{1-\frac{R}{r}}\left(\delta^{i j}-\frac{x^{i} x^{j}}{r^{3}} R\right), \quad x^{i}=x, y, z$.

Returning to polar coordinates we find (details are given in the Appendix A)

$$
\left.\epsilon^{i j}\right|_{\text {polar }}=\left.\mu^{i j}\right|_{\text {polar }}=\operatorname{diag} \epsilon_{0}\left[1, B^{-1}(r), B^{-1}(r)\right] .
$$

The subsequent wave equations are (full expressions are given in the Appendix A)

$$
\begin{gathered}
\epsilon_{0}^{2} \partial_{t}^{2} E_{r}-\nabla^{2} E_{r}=[F] \\
\frac{\epsilon_{0}^{2}}{B} \partial_{t}^{2} E_{\theta}-\nabla^{2} E_{\theta}=[G], \quad \frac{\epsilon_{0}^{2}}{B} \partial_{t}^{2} E_{\phi}-\nabla^{2} E_{\phi}=[H],
\end{gathered}
$$

where $F, G, H$ comprise of components of electric vector and their spatial derivatives. $E_{\theta}, E_{\phi}$ are linear combinations of our previous parameterization $E^{1}, E^{2}$. Notice that only the all important time derivative part of $E_{\theta}, E_{\phi}$ in Eq. (16) contains the horizon factor $B(r)$ and hence will participate in the quantum tunneling whereas $E_{r}$ in Eq. (15) will not contribute to tunneling which is somewhat counterintuitive.

\section{GRADED METAMATERIAL AND HAMILTON-JACOBI SCHEME}

Let us consider $\epsilon, \mu$ to be time-independent real functions of the form

$$
\epsilon(r)=\frac{\epsilon_{0}}{B(r)}, \quad \mu(r)=\frac{\mu_{0}}{B(r)},
$$

where $\epsilon_{0}, \mu_{0}$ denote constants (values at vacuum) with $c=1 / \sqrt{\left(\epsilon_{0} \mu_{0}\right)}$ denoting the velocity of electromagnetic wave in vacuum and $B(r)$ having a horizon at $r_{0}$ satisfying $B\left(r_{0}\right)=0$ with $B^{\prime}\left(r_{0}\right)$ being nonvanishing and finite.

The semiclassical or WKB wave function follows from making the standard ansatz

$$
E(r, t)=\exp \left[\frac{i}{\hbar} S(r, t)\right]
$$

Thus Eq. (B3) leads to the dynamical equation for $S$,

$$
\begin{aligned}
& \frac{1}{(c B)^{2}}\left(\frac{i}{\hbar} \ddot{S}-\frac{1}{\hbar^{2}}(\dot{S})^{2}\right)-\left(\frac{i}{\hbar} S^{\prime \prime}-\frac{1}{\hbar^{2}}\left(S^{\prime}\right)^{2}\right) \\
& -\frac{i}{\hbar} \rho(r) S^{\prime}-\sigma(r)=0
\end{aligned}
$$

where $\rho=\left(\frac{2}{r}-\frac{B^{\prime}}{B}\right), \sigma=\left[\left(\frac{B^{\prime}}{B}\right)^{2}-\frac{B^{\prime \prime}}{B}-\frac{2}{r^{2}}\right]$ and $\left.\dot{(}\right) \equiv \partial_{t}()$. As is customary [20], the expansion of $S$ in powers of $\hbar / i$, 


$$
S(r, t)=S_{0}(r, t)+\left(\frac{\hbar}{i}\right) S_{1}(r, t)+\left(\frac{\hbar}{i}\right)^{2} S_{2}(r, t)+\cdots
$$

is substituted in Eq. (B6) thereby yielding the lowest order in the $\hbar$ equation,

$$
\frac{E^{2}}{(c B)^{2}}-\left(S_{0}^{\prime}\right)^{2}+\hbar^{2} \sigma(r)=0
$$

In the above $E$ is a constant, the particle energy, obtained from the standard HJ identification $\dot{S}_{0}=-E$. Notice that, although of $O\left(\hbar^{2}\right)$, we have kept $\hbar^{2} \sigma(r)$ because it involves $1 / B(r)$ which is singular at the horizon. The solution of the $\mathrm{HJ}$ equation or equivalently the semiclassical wave function is derived as

$$
S_{0}(r, t)=-E t \pm \frac{1}{c} \int \frac{d r}{B} \sqrt{E^{2}-\hbar^{2} c^{2} B^{2} \sigma}
$$

Since $B^{2} \sigma$ is finite at the horizon we can safely ignore $\hbar^{2} B^{2} \sigma$ because in calculating Hawking effect the neighborhood of horizon is all that matters. Thus $S_{0}$ simplifies to

$$
S_{0}(r, t)=-E t \pm \frac{E}{c} \int \frac{d r}{B} .
$$

Before proceeding further, as is advocated in [20], we check the validity of the semiclassical ansatz by computing $S_{1}$ obtained from the next-to-leading order (in $\hbar$ ) equation from Eq. (B6),

$$
2\left(\frac{1}{(c B)^{2}} \dot{S}_{0} \dot{S}_{1}-S_{0}^{\prime} S_{1}^{\prime}\right)+\frac{1}{(c B)^{2}} \ddot{S}_{0}-S_{0}^{\prime \prime}-\rho S_{0}^{\prime}=0
$$

From Eq. (B10) we obtain

$$
\dot{S}_{0}=-E, \quad \ddot{S}_{0}=0, \quad S_{0}^{\prime}= \pm \frac{E}{c B}, \quad S_{0}^{\prime \prime}=\mp \frac{E B^{\prime}}{c B^{2}}=\mp \frac{E R}{c B^{2}}
$$

where near horizon $B(r) \approx B^{\prime}\left(r_{0}\right)\left(r-r_{0}\right) \equiv R\left(r-r_{0}\right)$. From Eq. (B11) we compute the $O(\hbar)$ correction to $S_{0}$, with $\dot{S}_{1}=-E_{1} t$,

$$
S_{1}(r, t)=-E_{1} t+\int d r\left( \pm \frac{E_{1}}{c B}+\frac{R}{2 B}+\frac{\rho}{2}\right)
$$

Since all the terms in the integrand have the same order of singularity at horizon as that of $S_{0}$, the validity of the semiclassical approximation is assured. We will be calculating the analogue Hawking temperature from Eq. (B10).

\section{EFFECTIVE SCHRÖDINGER PROBLEM}

It is also instructive to take a detour [20] to see that the above problem can be cast into a one-dimensional Schrödinger problem by making the substituting

$$
E(r, t)=\exp \left[\frac{i}{\hbar} E t\right] \frac{\sqrt{B}}{r} \Psi(r)
$$

in Eq. (B3). $\Psi$ satisfies a time-independent Schrödinger equation (in the limit $\hbar \rightarrow 0$ ),

$$
-\Psi^{\prime \prime}-\frac{g}{\left(r-r_{0}\right)^{2}} \Psi=0,
$$

where $g=(E /(c \hbar R))^{2}$. This equation is valid near the horizon with $\left(r-r_{0}\right)^{-2}$ dominating over other terms in the potential. We derive an inverted oscillator potential similar to [20]. To exploit the standard machinery the trick [20] is to introduce an energy $\tilde{E}$ by replacing Eq. (28) by

$$
-\Psi^{\prime \prime}-\frac{g}{\left(r-r_{0}\right)^{2}} \Psi=\tilde{E} \Psi
$$

and subsequently take the limit $\tilde{E} \rightarrow 0$. We will not repeat the steps of [20] which will yield the same result as Eq. (B10) that we will be following.

\section{ANALOGUE HAWKING TEMPERATURE AND ANALOGUE HAWKING RADIATION}

As we have emphasized throughout beauty and utility of the complex path formalism [20] is its generality. Operationally, we claim that since the relevant (or analogue) equations are structurally identical to the $\mathrm{BH}$ context similar conclusions should prevail. Again we will skip the detailed discussion provided in [20] and only present a few salient steps.

We will consider the function $B(r)$ such that it mimics the Schwarzschild-like metric where $B(r) \geq 0$ for $r \geq r_{0}$ and $B(r) \leq 0$ for $r \leq r_{0}$ with the horizon at $r=r_{0}$, $B\left(r_{0}\right)=0$. Thus in the disk shaped dielectric, the core region $r_{0} \geq r \geq 0$ will be of metamaterial type with negative dielectric parameters whereas the region $r \geq r_{0}$ will consist of normal dielectric. This will allow us to follow the same reasoning (as for Hawking radiation) where we treat the light going out from metamaterial to normal medium as outgoing and light from normal to metamaterial zone as incoming. The former and latter correspond to positive and negative sign respectively in the integral term of Eq. (B10). In fact the optical black hole laboratory system [11] is of this form.

Thus recalling Eq. (B10) for photons crossing the analogue horizon from the core $r \leq r_{0}$ to $r \geq r_{0}$ with $\left(\partial S_{0}\right) /(\partial r) \geq 0$ we have 


$$
\begin{aligned}
S_{\mathrm{out}}^{0} & =-\left.\frac{E_{1}}{c} \int_{r_{0}-\epsilon}^{r_{0}+\epsilon} \frac{d r}{B}\right|_{\epsilon \rightarrow 0}+(\text { real part }) \\
& =\frac{i \pi E}{R\left(r_{0}\right)}+(\text { real part })
\end{aligned}
$$

Similarly, for photons crossing the analogue horizon from outside $r \geq r_{0}$ in to the core $r \leq r_{0}$ with $\left(\partial S_{0}\right) /(\partial r) \leq 0$ we have

$$
\begin{aligned}
S_{\mathrm{in}}^{0} & =-\left.\frac{E_{1}}{c} \int_{r_{0}+\epsilon}^{r_{0}-\epsilon} \frac{d r}{B}\right|_{\epsilon \rightarrow 0}+(\text { real part }) \\
& =-\frac{i \pi E}{R\left(r_{0}\right)}+(\text { real part }) .
\end{aligned}
$$

The modulus square of the above expressions yields the respective probabilities,

$$
P[\text { out }] \infty \exp \left(-\frac{2 \pi E}{\hbar R}\right), \quad P[\text { in }] \infty \exp \left(\frac{2 \pi E}{\hbar R}\right),
$$

which in turn means that

$$
P[\text { out }]=\exp \left(-\frac{4 \pi E}{\hbar R}\right) P[\text { in }] .
$$

This is written as $P[o u t]=\exp (-\beta E) P[i n]$ with the analogue Hawking temperature of the horizon identified as

$$
\beta=\frac{1}{k T}=\frac{4 \pi}{c \hbar R},
$$

where $k$ is the Boltzmann constant. If we consider $B(r)$ of the Schwarzschild form $B(r)=1-r_{0} / r$ then the analogue Hawking temperature at $r=r_{0}$ becomes

$$
T=\frac{c \hbar}{4 \pi k r_{0}} .
$$

The above Eq. (34) constitutes our main result. Expressing in another way for $T \approx 1 \mathrm{~K}$ requires $r_{0} \sim 10^{-3}$ meter and $T \approx 300 \mathrm{~K}$ will need the composite disk to have $r_{0} \sim 3 \times 10^{-6}$ meter. A more realistic choice might be $B=b \tan h\left(\frac{r-r_{0}}{r_{0}}\right)$ where $b$ is a dimensionless constant. In this case $R=b / r_{0}$ yielding thereby

$$
T=b \frac{c \hbar}{4 \pi k r_{0}} .
$$

The possibility of experimental observation of Hawking effect from the disk-shaped metamaterial composite appears to be quite promising. The main challenge seems to be the dimension of the system since the analogue Hawking temperature $T \sim\left(r_{0}\right)^{-1}$, larger $T$ requires a smaller $r_{0}$, the analogue horizon. In the case of [11] the disk diameter is $\sim 10^{-3}$ meters.

\section{DISCUSSION}

In this paper we have shown the potential possibility of Hawking-like junction temperature and subsequent Hawking-like radiation from the junction of a composite normal-metamaterial dielectric. We have used the complex path formalism [17], closely following the work [20] as applied to Hawking radiation. We have exploited the generality involved in the formalism that requires only an effective horizonlike structure in the wave equation of the relevant degree of freedom involved. Intuitively one can understand the special status of the analogue horizon and the need to invoke the tunneling mechanism to cross the horizon in the following ways. On one hand, the effective (phase) velocity of the wave $\sim B(r) / \sqrt{\epsilon_{0} \mu_{0}}$ vanishes at the horizon. On the other hand, in the geometrical optics limit we have the relation $p=$ $\sqrt{\left(\epsilon(r) / \epsilon_{0}\right)} E / c=\sqrt{(1 / B(r))} E / c$ where (for the zero angular momentum case we are restricting to), $p$ and $E$ correspond to the photon momentum and energy (or frequency) respectively. Thus, outside the horizon $p$ becomes imaginary and classically forbidden and the photons have to tunnel out.

The ambient electromagnetic field energy will act as the source of this radiation. It should be noted that, in the present paper, we have not taken into account two very important aspects of metamaterial: it is inherently dispersive and lossy in nature. To address these issues, the dielectric parameters need to be frequency dependent and complex in nature. Hence our model and results are actually valid for a narrow range of frequency with negligible loss. We plan to return to these topics in the near future.

\section{ACKNOWLEDGMENTS}

We thank Ted Jacobson, S. Shankaranarayanan and Bibhas Ranjan Majhi for correspondence.

\section{APPENDIX A}

We derive the dielectric parameters corresponding to the Schwarzschild metric. The Schwarzschild metric in conventional polar form is given by

$$
\begin{aligned}
d S^{2}= & g_{\mu \nu} d x^{\mu} d x^{\nu}=\left(1-\frac{R}{r}\right) d t^{2}+\frac{d r^{2}}{\left(1-\frac{R}{r}\right)} \\
& +r^{2}\left(d \theta^{2}+\sin ^{2} \theta d \phi^{2}\right) .
\end{aligned}
$$

Permittivity and permeability, for a metamaterial, can be described by the metric components as 


$$
\epsilon^{i j}=\mu^{i j}=-\frac{\sqrt{-g}}{g_{00}} g^{i j},
$$

where this map is in the Cartesian coordinates system. Explicitly the Schwarzschild metric in Cartesian coordinates is written as

$$
\epsilon^{i j}=\mu^{i j}=-\frac{1}{1-\frac{R}{r}}\left(\delta^{i j}-\frac{x^{i} x^{j}}{r^{3}} R\right),
$$

where $x^{i}=x, y, z$. This leads to the dielectric parameters:

$$
\epsilon^{i j}=\mu^{i j}=\frac{1}{1-\frac{R}{r}}\left[\begin{array}{ccc}
-1+\frac{R x^{2}}{r^{3}} & x y \frac{R}{r^{3}} & x z \frac{R}{r^{3}} \\
x y \frac{R}{r^{3}} & -1+\frac{R y^{2}}{r^{3}} & y z \frac{R}{r^{3}} \\
x z \frac{R}{r^{3}} & y z \frac{R}{r^{3}} & -1+\frac{R z^{2}}{r^{3}}
\end{array}\right] .
$$

First we have to substitute $x, y, z$ in terms of polar coordinates $r, \theta, \phi$. Since we are interested in working in the spherical polar coordinate system, we exploit the transformation that converts the Cartesian tensor to a tensor in the polar coordinate:

$$
\begin{aligned}
& {\left[\begin{array}{ccc}
\epsilon^{r r} & \epsilon^{r \theta} & \epsilon^{r \phi} \\
\epsilon^{\theta r} & \epsilon^{\theta \theta} & \epsilon^{\theta \phi} \\
\epsilon^{\phi r} & \epsilon^{\phi \theta} & \epsilon^{\phi \phi}
\end{array}\right] } \\
&= {\left[\begin{array}{ccc}
\sin \theta \cos \phi & \sin \theta \sin \phi & \cos \theta \\
\cos \theta \cos \phi & \cos \theta \sin \phi & -\sin \theta \\
-\sin \phi & \cos \phi & 0
\end{array}\right] } \\
& \times {\left[\begin{array}{ccc}
\epsilon^{x x} & \epsilon^{x y} & \epsilon^{x z} \\
\epsilon^{y x} & \epsilon^{y y} & \epsilon^{y z} \\
\epsilon^{z x} & \epsilon^{z y} & \epsilon^{z z}
\end{array}\right] } \\
& \times {\left[\begin{array}{ccc}
\sin \theta \cos \phi & \cos \theta \cos \phi & -\sin \phi \\
\sin \theta \sin \phi & \cos \theta \sin \phi & \cos \phi \\
\cos \theta & -\sin \theta & 0
\end{array}\right] . }
\end{aligned}
$$

This yields $\epsilon^{i j}, \mu^{i j}$ in polar form,

$$
\epsilon^{i j}=\mu^{i j}=\frac{1}{1-\frac{R}{r}}\left[\begin{array}{ccc}
1-\frac{R}{r} & 0 & 0 \\
0 & 1 & 0 \\
0 & 0 & 1
\end{array}\right] \text {. }
$$

Defining $B(r)=1-\frac{R}{r}$, we get

$$
\epsilon^{i j}=\mu^{i j}=\left[\begin{array}{ccc}
\epsilon_{0} & 0 & 0 \\
0 & \frac{\epsilon_{0}}{B(r)} & 0 \\
0 & 0 & \frac{\epsilon_{0}}{B(r)}
\end{array}\right] .
$$

\section{APPENDIX B}

We derive the wave equation satisfied by electromagnetic fields in polar form from Maxwell's equations. The components of electric displacement are

$$
\begin{gathered}
D_{r}=\epsilon_{0} E_{r}, \quad D_{\theta}=\frac{\epsilon_{0}}{B(r)} E_{\theta}, \quad D_{\phi}=\frac{\epsilon_{0}}{B(r)} E_{\phi} \quad(\mathrm{B} 1) \\
\nabla . \mathbf{E}=\frac{1}{r}\left(1-\frac{1}{B}\right)\left[\partial_{\theta} E_{\theta}+\frac{\cos \theta}{\sin \theta} E_{\theta}+\frac{\partial_{\phi} E_{\phi}}{\sin \theta}\right] \quad(\mathrm{B} 2) \\
\nabla \times \mathbf{B}=\epsilon_{0}(\nabla \times \mathbf{H})+\frac{\epsilon_{0}}{r^{2} \sin \theta}\left[\hat { r } ( \frac { 1 } { B } - 1 ) \left\{\partial _ { \theta } \left(r \sin \theta H_{\phi}\right.\right.\right. \\
\left.-\partial_{\phi}\left(r H_{\theta}\right)\right\}+r \hat{\theta}\left\{-\left(\frac{1}{B}-1\right) \partial_{r}\left(r \sin \theta H_{\phi}\right)\right. \\
\left.-r \sin \theta h_{\phi} \partial_{r} \frac{1}{B}\right\} \\
\left.+r \sin \theta \hat{\phi}\left(\left(\frac{1}{B}-1\right) \partial_{r}\left(r^{2} H_{\theta}\right)+r^{2} H_{\theta} \partial_{r} \frac{1}{B}\right)\right] \\
(\nabla \times \mathbf{E})_{\hat{r}}=\frac{1}{r^{2} \sin \theta}\left[\partial_{\theta}\left(r \sin \theta E_{\phi}\right)-\partial_{\phi}\left(r E_{\theta}\right)\right]=-\epsilon_{0} \frac{\partial H_{r}}{\partial t} \\
(\mathrm{~B} 3) \\
\nabla \times \mathbf{E}=-\frac{\partial B}{\partial t}
\end{gathered}
$$

The wave equation is given by

$$
\nabla(\nabla . \mathbf{E})-\nabla^{2} \mathbf{E}=\frac{\partial}{\partial t}(\nabla \times \mathbf{B})
$$

Individual components satisfy the following wave equations:

$\hat{r}$ equation:

$$
\epsilon_{0}^{2} \partial_{t}^{2} E_{r}-\nabla^{2} E_{r}=[F]
$$

where 


$$
\begin{aligned}
{[F]=} & {\left[\frac { 1 - B } { r ^ { 2 } \operatorname { s i n } \theta } \left\{\left(\partial_{\theta}^{2}+\frac{1}{\sin \theta} \partial_{\phi}^{2}\right) E_{r}\right.\right.} \\
& \left.\left.-\partial_{\theta}\left(\sin \theta\left(E_{\theta}+r \partial_{r} E_{\theta}\right)\right)-\partial_{\phi}\left(E_{\phi}+r \partial_{r} E_{\phi}\right)\right\}\right] \\
& -\frac{\partial}{\partial r}\left[\frac{B-1}{r B}\left\{\partial_{\theta} E_{\theta}+\frac{\cos \theta}{\sin \theta} E_{\theta} \frac{1}{\sin \theta} \partial_{\phi} E_{\phi}\right\}\right]
\end{aligned}
$$

$\hat{\theta}$ equation:

$$
\frac{\epsilon_{0}^{2}}{B} \partial_{t}^{2} E_{\theta}-\nabla^{2} E_{\theta}=[G]
$$

where

$$
\begin{aligned}
{[G]=} & -\frac{1}{r}\left\{(1-B) \partial_{r}-\partial_{r} B\right\} \times\left\{E_{\theta}+r \partial_{r} E_{\theta}-\partial_{\theta} E_{r}\right\} \\
& -\frac{1}{r} \frac{\partial}{\partial \theta}\left[\frac{B-1}{r B}\left(\partial_{\theta} E_{\theta}\right)+\frac{\cos \theta}{\sin \theta} E_{\theta} \frac{1}{\sin \theta} \partial_{\phi} E_{\phi}\right] ;
\end{aligned}
$$

$\hat{\phi}$ equation:

$$
\frac{\epsilon_{0}^{2}}{B} \partial_{t}^{2} E_{\phi}-\nabla^{2} E_{\phi}=[H],
$$

where

$$
\begin{aligned}
{[H]=} & -\frac{1}{r^{2}}\left\{-\frac{1-B}{\sin \theta} \partial_{r}+\frac{r}{\sin \theta} \partial_{r} B-\frac{1-B}{\sin \theta} r \partial_{r}\right\} \\
& \times\left\{\partial_{\phi} E_{r}-\sin \theta E_{\phi}-\sin \theta r \partial_{r} E_{\phi}\right\} \\
& -\frac{1}{r \sin \theta} \frac{\partial}{\partial \phi}\left[\frac{B-1}{r B}\left(\partial_{\theta} E_{\theta}\right)+\frac{\cos \theta}{\sin \theta} E_{\theta} \frac{1}{\sin \theta} \partial_{\phi} E_{\phi}\right] .
\end{aligned}
$$

[1] S. W. Hawking, Black hole explosions?, Nature (London) 248, 30 (1974).

[2] W. G. Unruh, Experimental Black-Hole Evaporation?, Phys. Rev. Lett. 46, 1351 (1981).

[3] C. Barcelo, S. Liberati, and M. Visser, Analogue gravity, Living Rev. Relativity 8, 12 (2005).

[4] S. Weinfurtner, E. W. Tedford, M. C. J. Penrice, W. G. Unruh, and G. A. Lawrence, Measurement of Stimulated Hawking Emission in an Analogue System, Phys. Rev. Lett. 106, 021302 (2011).

[5] W. Gordon, Zur Lichtfortpflanzung nach der Relativitätstheorie, Ann. Phys. (Leipzig) 72, 421 (1923); J. Plebanski, Electromagnetic waves in gravitational fields, Phys. Rev. 118, 1396 (1960); L. Landau and E. M. Lifshitz, The Classical Theory of Fields (Elsevier, Oxford, 2000).

[6] V. G. Veselago, The electrodynamics of substances with simultaneously negative values of $\epsilon$ and $\mu$, Sov. Phys. Usp. 10(4), 509 (1968); V. G. Veselago, L. Braginsky, V. Shk Lover, and C. Hafner, Negative refractive index materials, J. Comput. Theor. Nanosci. 3, 189 (2006).

[7] D. R. Smith, W. J. Padilla, D. C. Vier, S. C. Nemat-Nasser, and S. Schultz, Composite Medium with Simultaneously Negative Permeability and Permittivity, Phys. Rev. Lett. 84, 4184 (2000); R. A. Shelby, D. R. Smith, and S. Schultz, Experimental verification of a negative index of refraction, Science 292, 77 (2001); S. Linden, C. Enkrich, M. Wegener, J. Zhou, T. Koschny, and C. M. Soukoulis, Magnetic response of metamaterials at 100 Terahertz, Science 306, 1351 (2004); V. M. Shalaev, W. Cai, U. K. Chettiar, H. Yuan, A. K. Sarychev, V. P. Drachev, and A. V. Kildishev, Negative index of refraction in optical metamaterials, Opt. Lett. 30, 3356 (2005); W. J. Padilla, D. N. Basov, and D. R. Smith, Negative refractive index metamaterials, Mater. Today 9, 28 (2006); P. Lähteenmäki, G. S. Paraoanu, J. Hassel, and P. J. Hakonen, Dynamical Casimir effect in a Josephson metamaterial, Proc. Natl. Acad. Sci. U.S.A. 110, 4234 (2013).

[8] Y. Liu and X. Zhang, Metamaterials: A new frontier of science and technology, Chem. Soc. Rev. 40 (2011); G. Singh, R. Rajni, and A. Marwaha, Modeling of split ring resonators loaded microstrip line with different orientations, Int. J. Eng. Trends Technol. 19, 1 (2015).

[9] I. I. Smolyaninov, Hyperbolic metamaterials, arXiv:1510 .07137; Surface plasmon toy model of a rotating black hole, New J. Phys. 5, 147 (2003); Quantum electromagnetic "black holes" in a strong magnetic field, J. Phys. G 40, 015005 (2013).

[10] E. E. Narimanov and A. V. Kildishev, Optical black hole: Broadband omnidirectional light absorber, Appl. Phys. Lett. 95, 041106 (2009).

[11] Q. Cheng, T. J. Cui, W.X. Jiang, and B. G. Cai, An omnidirectional electromagnetic absorber made of metamaterials, New J. Phys. 12, 063006 (2010).

[12] S. Dehdashti, H. Wang, Y. Jiang, Z. Xu, and H. Chen, Review of black hole realization in laboratory based on transformation optics, Prog. Electromagn. Res. 154, 181193 (2015); W. Zhu, Electromagnetic metamaterial absorbers: From narrowband to broadband, metamaterials and metasurfaces, Intech Open, https://doi.org/10.5772/intechopen.78581.

[13] S. Ghosh and S. K. Maiti, Spontaneous photoemission from metamaterial junction: A conjecture, arXiv:1306.3748.

[14] B. Reznik, Origin of the thermal radiation in a solidstate analogue of a black hole, Phys. Rev. D 62, 044044 (2000). 
[15] R. Schutzhold, G. Plunien, and G. Soff, Quantum radiation in external background fields, Phys. Rev. A 58, 1783 (1998).

[16] F. Belgiorno, S. L. Cacciatori, G. Ortenzi, V. G. Sala, and D. Faccio, Quantum Radiation from Superluminal Refractive-Index Perturbations, Phys. Rev. Lett. 104, 140403 (2010).

[17] L. D. Landau and E. M. Lifshitz, Quantum Mechanics (Nonrelativistic Theory), Course of Theoretical Physics Vol. 2 (Pergamon Press, New York, 1975).

[18] M. K. Parikh and F. Wilczek, Hawking Radiation as Tunneling, Phys. Rev. Lett. 85, 5042 (2000); R. Banerjee and B.R. Majhi, Quantum tunneling and back reaction, Phys. Lett. B 662, 62 (2008).
[19] S. P. Robinson and F. Wilczek, Relationship between Hawking Radiation and Gravitational Anomalies, Phys. Rev. Lett. 95, 011303 (2005); R. Banerjee and B. R. Majhi, Connecting anomaly and tunneling methods for Hawking effect through chirality, Phys. Rev. D 79, 064024 (2009); S. Ghosh, Combining the tunneling and anomaly phenomena in deriving the gravitational anomaly, Mod. Phys. Lett. A 24, 2639 (2009).

[20] K. Srinivasan and T. Padmanabhan, Particle production and complex path analysis, Phys. Rev. D 60, 024007 (1999); see also S. Shankaranarayanan, Temperature and entropy of Schwarzschild-de Sitter space-time, Phys. Rev. D 67, 084026 (2003). 